\title{
Anesthetic experience of a patient with severe change on respiratory mechanics in the prone position for spinal surgery
}

\author{
Soo Kyung Lee, Min Chul Kim, Yi-Hwa Choi, Mae Hwa Kang, and EunYoung Park \\ Department of Anesthesiology and Pain Medicine, Hallym University Sacred Heart Hospital, Hallym University College of Medicine, \\ Anyang, Korea
}

Prone positioning has commonly been used for posterior spinal surgery. However, it has the potential to increase intraabdominal pressure (IAP), and adversely affects cardiovascular and pulmonary functions [1]. We describe a patient who developed severe deterioration of respiratory mechanics during anesthesia for spinal surgery in the prone position.

A 64-year-old man (172 cm of height, $78 \mathrm{~kg}$ of weight) was presented for decompressive laminectomy L4-5 caused by spinal stenosis. He had hypertension, hypercholesterolemia, and multiple-vessel coronary artery disease, and had undergone a percutaneous coronary intervention on mid-right coronary artery (RCA) eight years ago. A transthoracic echocardiogram showed ischemic insult at RCA territory and preserved left ventricular systolic function. Anesthesia induction and endotracheal intubation were achieved using propofol, remifentanil, and rocuronium with a $7.5 \mathrm{~mm}$ reinforced tube. Anesthesia was maintained with sevoflurane, remifentanil, and 50\% oxygen in air. Mechanical ventilation was adjusted with a tidal volume of $600 \mathrm{ml}$ and respiratory rate of 12 times/min, and peak inspiratory pressure (PIP) was $17 \mathrm{cmH}_{2} \mathrm{O}$ in the supine position. The patient's position was changed to prone on the Wilson frame after radial artery cannulation. The surgeon lifted the Wilson frame fully and placed pads beneath the iliac crest to decrease lordosis of the lumbar spine. At that time, PIP was about $21 \mathrm{~cm}$ $\mathrm{H}_{2} \mathrm{O}$. After about 30 minutes, PIP increased to $30 \mathrm{cmH}_{2} \mathrm{O}$. The patient's lung sound was clear, but decreased on both lung fields. Wheezing or rale was unremarkable. Although the possibility of reactive airway disease was low, we increased the concentration of sevoflurane and administered salbutamol inhaler $(0.1 \mathrm{mg})$ via endotracheal tube 2 times to rule out bronchial asthma. PIP further increased to $33 \mathrm{mmHg}$ even after administration of additional salbutamol inhaler and intravenous steroid. We changed the mode of the ventilator from volume controlled ventilation to pressure controlled ventilation with PIP of $26 \mathrm{cmH}_{2} \mathrm{O}$ and a respiratory rate of 14 times/min, and delivered tidal volume was $270 \mathrm{ml}$. Other causes of increased airway pressure such as secretion or endobronchial intubation could be excluded through suctioning and auscultation. We increased the PIP to 28 $\mathrm{cmH}_{2} \mathrm{O}$, but delivered tidal volume gradually decreased to less than $200 \mathrm{ml}$ although $\mathrm{SpO}_{2}$ was maintained to $98 \%$ with $\mathrm{FIO}_{2}$ of 0.65 . We suspected an acute rise in IAP and further increases or long-lasting intra-abdominal hypertension (IAH) might result in hypoxia and hypotension. So we changed patient's position to supine and ventilator mode to volume controlled ventilation simultaneously. PIP decreased abruptly to less than $20 \mathrm{cmH}_{2} \mathrm{O}$ with a tidal volume of $500 \mathrm{ml}$. The Wilson frame was changed to a Relton and Hall frame, and the patient was repositioned. Thereafter, the patient's PIPs maintained at $19-20 \mathrm{cmH}_{2} 0$ with a tidal volume of $600 \mathrm{ml}$ during the intraoperative period and the surgical procedure was uneventful.

Prone positioning may be associated with an increased IAP and abdominothoracic transmission during IAH influences on multiple organ systems. Obstruction of the inferior vena cava, occurring in the prone position, is exaggerated by abdominal compression, and results in reduction of cardiac output [1]. IAH was defined as an IAP at or above $12 \mathrm{mmHg}$, and risk fac-

Corresponding author: Soo Kyung Lee, M.D., Ph.D., Department of Anesthesiology and Pain Medicine, Hallym University Sacred Heart Hospital, 22, Gwanpyeong-ro, 107 beon-gil, Dongan-gu, Anyang 431-796, Korea. Tel: 82-31-380-3945, Fax: 82-31-385-3244, E-mail: agnetask@naver.com (c) This is an open-access article distributed under the terms of the Creative Commons Attribution Non-Commercial License (http:// creativecommons.org/licenses/by-nc/3.0/), which permits unrestricted non-commercial use, distribution, and reproduction in any medium, provided the original work is properly cited. 
tors include mechanical ventilation, pneumoperitoneum, tight abdominal closures, and prone positioning. The transmission of IAP to the thorax can influence the respiratory system, reducing the functional residual capacity, chest wall compliance, and overall compliance of the respiratory system [2]. Progressive elevation of IAP leads to hypoventilation associated with increased pulmonary vascular resistance because of reduction in alveolar oxygen tension and elevation of intrathoracic pressure. It is manifested by hypercapnia, elevated ventilator pressure, and hypoxia [3].

There are many positioning devices and positions for use in spinal surgery, which serve to suspend the abdomen. The Wilson frame is commonly used in spinal surgery. It is a convenient device having two curved full-length pads that maintained the patient in a flexed position. Lateral adjustment of the pads helps improve ventilation and alleviate pressure from the abdomen [4]. However, one study reported that IAP in the prone position on the Wilson frame with narrow pad width was significantly greater than in the supine position after induction, but not on wide support. It was suggested that flexed pads in narrow support could compress the abdomen of patient rather than relieving abdominal compression [5]. In the present case, the patient was positioned on the fully lifted and relatively narrow support Wilson frame. Although acute and severe increase in IAP in patients positioned on the Wilson frame is rare, it is possible to result in severe deterioration of mechanical ventilation as in the present case. However, respiratory mechanics recovered to the initial and acceptable state following the position change to supine. After repositioning on the Relton and Hall frame, which consisted of four robust pad supports arranged in two V-shaped pairs [4], anesthetic managements occurred without any events. Therefore, it is necessary to consider repositioning of the patient, by means of changing the support frame or widening the pad support, to reduce the alterations in physiologic changes.

Certain surgeons prefer the fully flexed Wilson frame to decrease lordosis of the lumbar spine and facilitate surgical exposure in posterior spinal surgery. However, a fully lifted narrow frame could result in compression of the abdomen and increased IAP. In the present case, the surgeon placed additional pads beneath the iliac crest to induce lumbar kyphosis. These situations could aggravate elevation of IAP. It is desirable that the width between pad supports of the Wilson frame be as broad as possible to reduce IAP accompanying with prevention of downward migration of the patient between the pads [5]. Therefore, it is necessary to create a balance between abdominal decompression and lordosis preservation in the prone position on the surgical frames.

In conclusion, the prone positioning and support system used affects the physiologic changes of various organ systems. Anesthesiologists should be aware that the prone positioning has the potential to induce IAH, and abdominothoracic transmission has an impact on cardiovascular as well as pulmonary function. Special attention should be paid to correct prone positioning in order to create both adequate surgical condition and minimal physiologic changes.

\section{References}

1. Edgcombe H, Carter K, Yarrow S. Anaesthesia in the prone position. Br J Anaesth 2008; 100: 165-83.

2. de Laet IE, Malbrain M. Current insights in intra-abdominal hypertension and abdominal compartment syndrome. Med Intensiva 2007; 31 : 88-99.

3. Lerner SM. Review article: the abdominal compartment syndrome. Aliment Pharmacol Ther 2008; 28: 377-84.

4. Schonauer C, Bocchetti A, Barbagallo G, Albanese V, Moraci A. Positioning on surgical table. Eur Spine J 2004; 13 Suppl 1: S50-5.

5. Park CK. The effect of patient positioning on intraabdominal pressure and blood loss in spinal surgery. Anesth Analg 2000; 91: 552-7. 